\title{
Perspective
}

Actualité en histoire de l'art

\section{Entretien avec Roland Recht}

par Laurent Baridon, Agnès Callu et François-René Martin

Roland Recht, Laurent Baridon, Agnès Callu et François-René Martin

\section{CpenEdition}

Journals

Édition électronique

URL : https://journals.openedition.org/perspective/7103

DOI : $10.4000 /$ perspective. 7103

ISSN : 2269-7721

Éditeur

Institut national d'histoire de l'art

Édition imprimée

Date de publication : 30 juin 2017

Pagination : 47-64

ISBN : 9782917902387

ISSN : $1777-7852$

Référence électronique

Roland Recht, Laurent Baridon, Agnès Callu et François-René Martin, «Entretien avec Roland Recht », Perspective [En ligne], 1 | 2017, mis en ligne le 31 décembre 2017, consulté le 16 janvier 2023. URL: http://journals.openedition.org/perspective/7103; DOI : https://doi.org/10.4000/perspective.7103 


\title{
Entretien avec Roland Recht
}

\author{
par Laurent Baridon, Agnès Callu \\ et François-René Martin
}

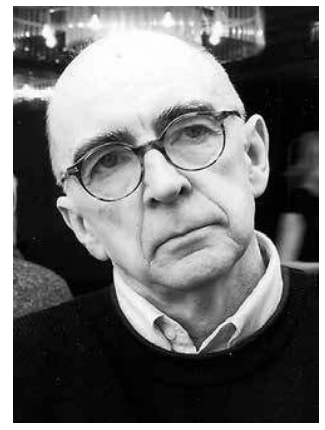

L'idée de l'entretien est ici, sinon biaisée, contournée, mais en faveur d'un résultat singulier quand, au lieu de restituer une seule trajectoire intellectuelle, il se propose à la façon d'une "conversation entre amis". De fait, quadrilogue composé et organisé autour de l'historien de l'art Roland Recht, professeur honoraire au Collège de France et membre de l'Académie des Inscriptions et Belles-Lettres de l'Institut de France, il réunit Laurent Baridon, historien de l'art, professeur à l'université Lyon 2, Agnès Callu, historienne, conservateur du Patrimoine au musée des Arts décoratifs, chercheur associé permanent au CNRS (IHTP) et François-René Martin, historien de l'art, professeur à l'École nationale supérieure des Beaux-Arts (Paris), chercheur invité au Centre allemand d'histoire de l'art.

Cet entretien capture l'amical respect de trois historiens pour un "maître", admiré mais toujours demeuré accessible car soucieux de transmission, simple de contact, ouvert aux débats, prescripteur dans l'écriture d'une histoire de l'art in progress ${ }^{l}$. Roland Recht choisit celle et ceux qui le questionnent, voulant rassembler autour de lui non seulement des amis fidèles, mais sans doute, aussi, un groupe soudé par des affinités électives manifestes : ses élèves le composent, à Dijon, pour Laurent Baridon ${ }^{2}$ à Strasbourg, pour François-René Martin', avec Agnès Callu, rencontrée en 2013 lors d'un séminaire doctoral de l'EPHE consacré à une anthropologie du musée contemporain ${ }^{4}$.

À l'occasion d'une journée passée, en octobre 2016, près de Strasbourg, dans la maison de Roland Recht, l'entretien s'élabore à la manière de lianes discursives, dans une volonté de novation : ni l'ego-histoire, bientôt restituée dans les pages d'un volume d'entretiens, ${ }^{5}$ ni la répétition de dialogues très spécialisés sur des sujets experts ${ }^{6}$. La relation d'un débat d'idées où interviennent l'art, la politique ou l'historiographie est davantage recherchée. Sans censure et au rythme des questions de chacun, présentées selon des lexiques communs ou non, des interprétations, divergentes ou non, des points d'appuis, personnels ou non, s'élabore une manière de "retex", un "retour d'expérience " de Roland Recht où le récit intellectuel s'avance à la jointure de la narration, du discours et de l'événement, sur une histoire de l'art au présent, lorsque ce dernier est compris autant comme la mémoire des choses passées que l'attente des choses à venir, pour citer saint Augustin.

Une partie des échanges a concerné l'histoire de l'histoire de l'art. On sait l'importance qu'ont pu prendre les recherches dans ce domaine depuis plus de vingt-cinq ans, en France moins qu'à l'étranger. Roland Recht fut l'un des principaux acteurs 


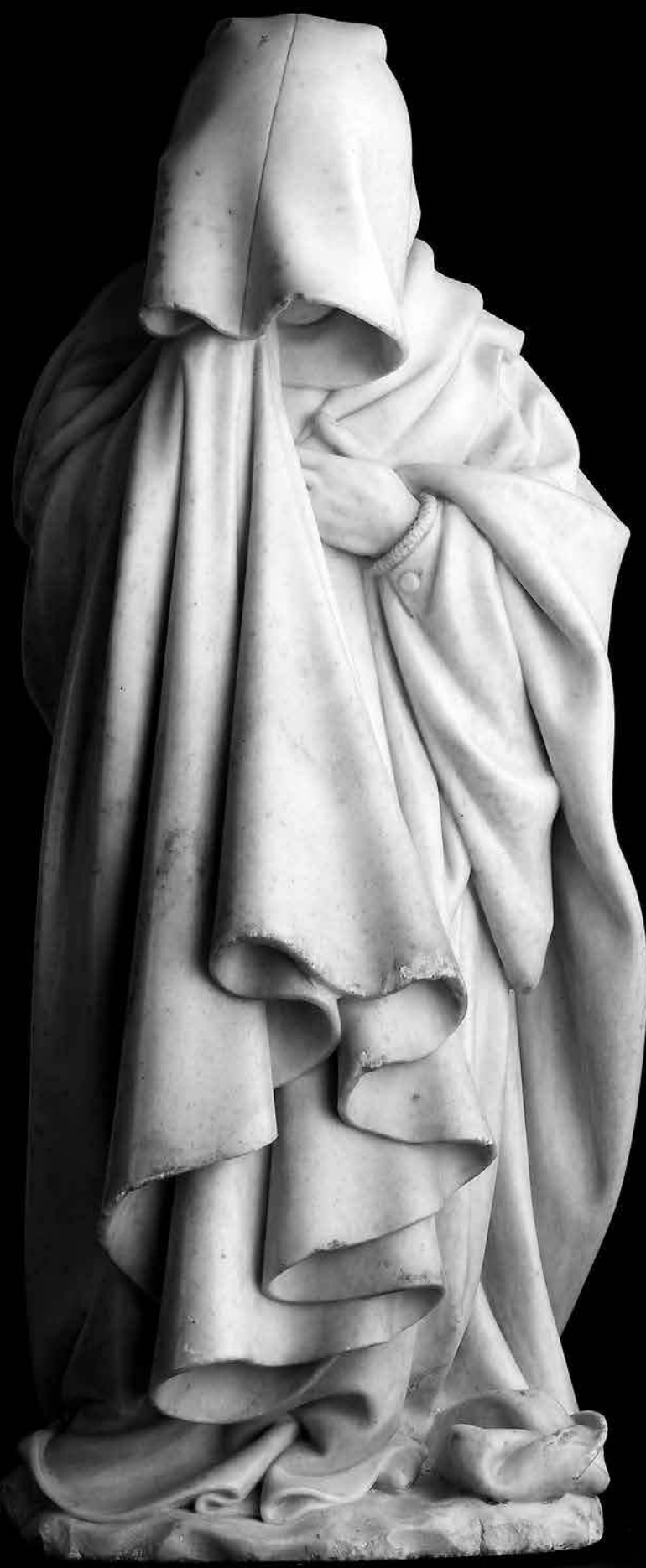


de ce tournant réflexif en France, dans un dialogue critique avec quelques-uns de ses homologues allemands ou italiens ${ }^{7}$. Tout travail d'ampleur en histoire de l'art implique une traversée du domaine historiographique - laquelle s'exprime à la fois dans le tissu des travaux "classiques " sur les auvres, les courants artistiques, etc., et dans ceux qui concernent directement le domaine historiographique. Il faut à la fois lire la thèse de Roland Recht sur Nicolas de Leyde, ses essais sur Claus Sluter (fig. 1) ou Auguste Rodin, où l'on sent bien la dette contractée auprès de l'un de ses grands prédécesseurs dans les études sur la sculpture, Wilhelm Vöge, et ses travaux sur les grandes figures de la Kunstwissenschaft ${ }^{9}$, pour bien comprendre que l'histoire de l'histoire de l'art, qui est certes une histoire des idées, doit être pratiquée sans jamais perdre le rapport aux auvres qui lui donne tout son sens. Leçon sur le passé de la discipline, sur les rendez-vous manqués ou les malentendus dans le cas de la France, l'histoire de l'histoire de l'art délivre également une leçon civique ou, plus largement, politique. De tout cela il fut question dans nos entretiens, et les éclairages que Roland Recht donne en la matière concernent autant le monde savant que la sphère publique, où s'entrecroisent le monde des musées et du patrimoine, où s'établissent les politiques culturelles ou le rapport à la mémoire collective.

Roland Recht évoque ensuite ses travaux en histoire de l'architecture. Il est nécessaire de rappeler que c'est sous l'influence de son professeur Louis Grodecki que le jeune étudiant strasbourgeois se tourne vers l'étude de

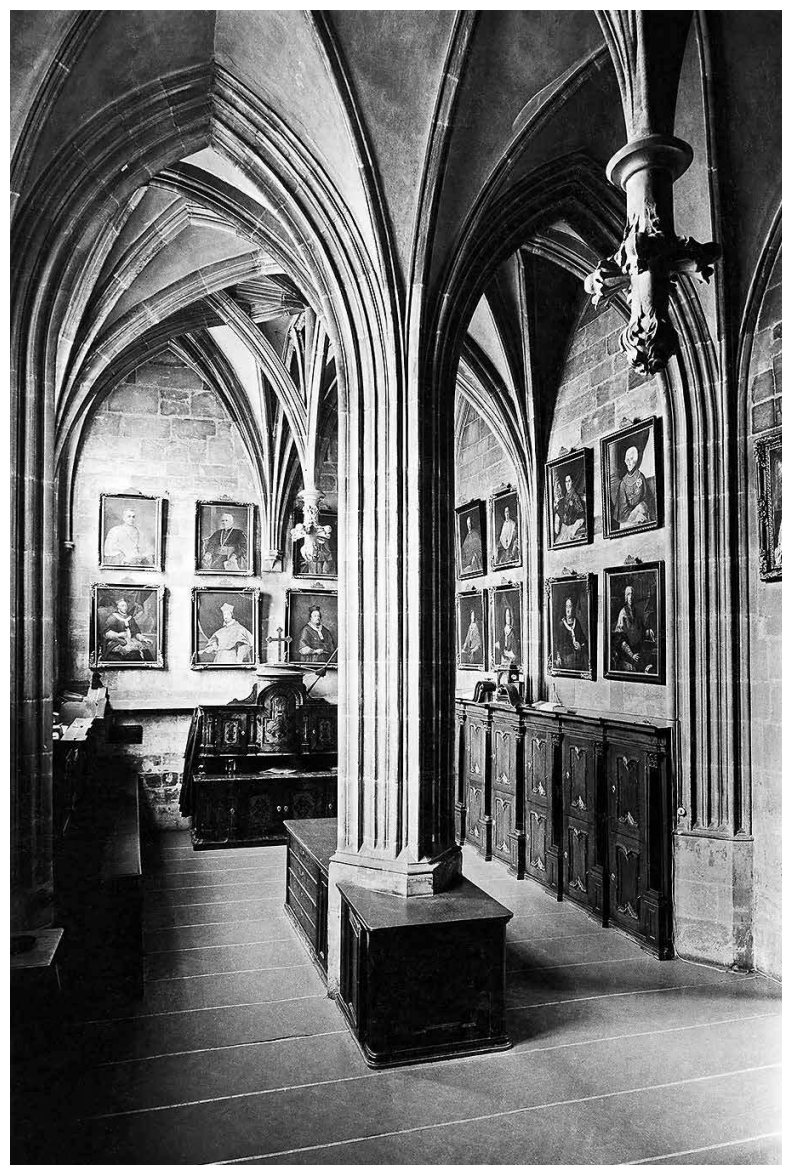
l'architecture religieuse médiévale dans le Rhin supérieur, et ses relations avec l'Europe centrale (fig. 2), un domaine qu'il n'abandonna jamais tout à fait. Néanmoins ses intérêts englobent à la fois la conception et la réalisation des édifices. C'est pourquoi les chantiers médiévaux et le dessin d'architecture ont retenu son attention ${ }^{10}$. Si Roland Recht est depuis longtemps internationalement connu pour ses travaux sur l'art médiéval, il est important de souligner que l'architecture a d'abord été une approche de l'art fondée sur l'espace. Pour expliciter la façon dont la notion d'espace, liée à celle de la perception des volumes et des formes, travaille l'approche de Roland Recht, il est utile de prendre un exemple en forme de souvenir d'étudiant : un cours sur Andrea Palladio ${ }^{11}$. Rétrospectivement, avec le recul de l'expérience, il est possible de comprendre ce qui le rendait si passionnant et formateur pour comprendre l'espace architectural : l'approche monographique traditionnelle était enrichie d'interprétations diverses, sociologiques par exemple, et d'ouvertures vers des questions contiguës - la tradition théorique, la représentation d'architecture - mais aussi vers des questions plus distantes - le paysage visible depuis les villas comme dans les villas, par les fresques qui les décorent, ainsi que la scénographie urbaine du Teatro Olimpico de Vicence. Initiation suggestive à la dimension spatiale de l'architecture et à son impossible transcription graphique, cet enseignement ouvrait vers l'image et le paysage. Il présageait d'autres études qui lient l'espace, l'image et le regard: autour de la question du paysage et de la photographie ${ }^{12}$; de l'architecture religieuse médiévale redéfinie en termes d'espace et de dispositif visuel ${ }^{13}$; de l'espace de l'auvre chez les artistes contemporains $^{14}$; ou encore, mais sans en publier les résultats, de la transparence de l'architecture

1. Claus Sluter, Claus de Werve, Pleurant du tombeau de Philippe le Hardi, Dijon, musée des Beaux-Arts, inv. CA 1416 n 21.

2. Sacristie de la cathédrale Saint-Guy, Prague. 
contemporaine et des dispositifs virtuels qui augmentent le regard et nous forcent à repenser la perception de l'espace par l'image.

[L'introduction et les questions sont composées par Laurent Baridon, Agnès Callu et François-René Martin]

Nos échanges se déroulent chez vous, en Alsace, à Niederhaslach. Votre vie d'historien de l'art et une part essentielle de votre carrière se sont déroulées dans une grande intimité avec la culture et l'art allemands. L'Alsace est une région qui ne pouvait que favoriser une telle immersion, dans deux cultures. Pouvez-vous revenir sur cet itinéraire, entre deux traditions historiographiques, et sur ce qu'un tel double point de vue autorise?

Les historiens de l'art français ont rarement discuté l'intitulé de leur discipline, contrairement aux Allemands. Le poids de la discipline historique, dans notre pays, est sans doute un élément d'explication. Faudrait-il ouvrir, en France, une discussion, qui traverse depuis plus d'un siècle la discipline en Allemagne, ou pouvons-nous nous contenter d'y rester extérieur?

Roland Recht. C'est l'immersion dont vous parlez qui a très tôt suscité un problème historiographique. Mes premiers travaux, vers 1965, ont porté sur des monuments d'une région, le Rhin supérieur, qui formait au Moyen Âge un diocèse réunissant les deux rives du fleuve, mais l'Alsace fit l'objet de revendications nationales comme on sait. La bibliographie dont je disposais était, par la force des choses, presque exclusivement en allemand, écrite par des historiens de l'art qui avaient enseigné à Strasbourg durant I'annexion à l'Empire (Georg Dehio ou Ernst Polaczek, par exemple), et encore bien après 1920, l'étude de l'art et de l'architecture du Moyen Âge sera laissée aux Allemands, les professeurs nommés à l'université de Strasbourg après le retour à la France n'étant pas des spécialistes d'architecture et, de surcroit, n'étant pas souvent germanophones. Je rencontrais au cours de ces travaux, d'une façon récurrente, des inflexions nettement partisanes qui tendaient ostensiblement à considérer l'art médiéval de cette région comme exclusivement tributaire de celui de l'Empire. Les éléments qui pouvaient parfois paraître indiscutables dans des analyses précises étaient rendus suspects par leur caractère systématique ou par une référence plus ou moins explicite au " génie allemand ». Au lendemain de la seconde guerre mondiale, l'Alsace était devenue un territoire annexé par l'historiographie allemande, en particulier par des chercheurs formés à l'université de Fribourg-en-Brisgau. Lorsque Willibald Sauerländer a commencé à enseigner à Fribourg, à partir de 1962-1963, il a aussitôt établi des relations avec son ami Louis Grodecki, professeur à Strasbourg, et un gentlemen's agreement a permis de mettre un

3. Quelques lectures de l'auteur dans les années 1960.

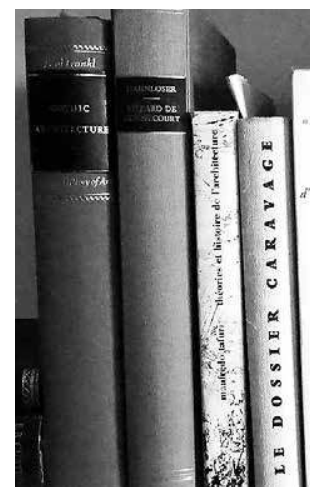
terme à cette mainmise systématique des thésards allemands sur l'art de la rive gauche du Rhin. Aux yeux de Grodecki, un Alsacien de ma génération offrait une garantie d'impartialité dans l'approche de ces monuments. Voilà comment, sur le terrain, est né chez moi l'intérêt pour l'historiographie ${ }^{15}$ (fig. 3).

Pour répondre à la deuxième partie de votre question, I'histoire de l'histoire de l'art est une branche de l'histoire de l'art en Allemagne depuis longtemps : la monographie de Carl Justi sur Winckelmann, le livre de Wilhelm Waetzoldt sur les historiens de l'art allemands ${ }^{16}$, sont des classiques. Cet intérêt va de pair avec un goût pour la théorie et la méthodologie ${ }^{17}$ et, depuis les années 1970 , I'historiographie s'est enrichie de nombreux travaux et en particulier de monographies d'historiens de l'art ${ }^{18}$. 
On assiste aujourd'hui en France à un intérêt grandissant pour l'historiographie de l'art mais il lui faut impérativement intégrer les grandes avancées qu'elle a connues de l'autre côté du Rhin. Or, ceci n'est possible qu'en passant par une meilleure connaissance de la langue. Les historiens de l'art français ignorent la plupart du temps les publications en langue allemande, préférant recourir, lorsqu'elles existent, à leurs traductions en italien ou en anglais. Il faut reconnaitre aussi que peu de collègues allemands sont au fait des travaux parus en France. II nous manque des " passeurs " comme l'ont été à différents titres Henri Berr, Maurice Halbwachs ou Raymond Aron. C'est ainsi que nous avons entrepris, avec Maurizio Ghelardi, de publier en France des écrits, parfois même inédits en allemand, d'Aby Warburg ${ }^{19}$. Ils conduiront peu à peu à une révision historiographique de première importance.

Pour qui vous a lu, ou a suivi votre enseignement, l'historiographie est un fil conducteur dans votre travail, chose rare dans notre discipline, tout au moins en France, où ce type de questionnement et de posture ne constitue pas un impératif. Comment expliquer ce déficit, ou ce déni - qui se double souvent d'un déni politique?

Roland Recht. La singularité de la situation française me semble devoir être examinée sous deux angles de vue. Tout d'abord, elle ignore le caractère spéculatif d'une bonne partie des travaux historiques, de l'intérêt marqué pour l'épistémologie des travaux historiques qui se manifestent en Allemagne dès le romantisme. Ce n'est pas un hasard si on y pratique deux disciplines, jumelles certes, mais qui ne se confondent pas : la Kunstgeschichte et la Kunstwissenschaft - histoire de l'art et science de l'art - la seconde se trouvant associée souvent à l'esthétique ou à la philosophie de l'art. Chez nous, il n'y a pas l'équivalent de la Kunstwissenschaft : elle est proche de ce que certains nomment aujourd'hui la « théorie de l'art ».

L'autre aspect qu'il convient de noter, c'est la place singulière qu'occupe I'histoire de l'art en France. Au XIXe siècle, les grands débats qui traversent la science historique effleurent à peine l'histoire de l'art ; son enseignement est peu répandu, manquant du socle théorique qu'avait bâti un Wilhelm von Humboldt à Berlin. Le second moment d'un tournant épistémologique manqué est celui de $\mathrm{I}^{\prime}$ « école » des Annales. Lorsqu'ils évoquent les sources non-écrites dont l'historien doit se servir, les «traces » comme ils les nomment, Marc Bloch et Lucien Febvre mentionnent I'archéologie mais pas l'art, ni la peinture ou l'architecture ${ }^{20}$. II me semble que cette amnésie, regrettable pour des historiens de cette envergure, doit être en grande partie imputée à l'hostilité que leur inspiraient l'œuvre et l'influence de Henri Focillon qui, ne l'oublions pas, domine alors la discipline du haut de sa chaire en Sorbonne. Son formalisme n'entrait pas dans les catégories des deux éminents historiens... Cette hostilité va se prolonger jusque bien après la guerre ${ }^{21}$, cette fois sous la forme d'un clivage entre une histoire de l'art sociologique (Francastel) et une autre, iconographique ou formaliste. Mais je voudrais souligner que, d'une certaine façon, les Annales ont ignoré l'apport de l'herméneutique ainsi qu'une partie du débat épistémologique autour de l'histoire comme science tel que l'historisme l'avait d'abord nourri en Allemagne, même si plusieurs définitions que donne en particulier Bloch sont très proches de celles d'un Droysen ${ }^{22}$.

À mes yeux, l'historiographie fait partie intégrante de la discipline et l'absence de débat théorique est une grande lacune en France ${ }^{23}$. En même temps, je la définirai comme un champ de recherche large qui ne peut pas se contenter de citer des extraits d'ouvrages savants de tel ou tel auteur. Par exemple, dans un essai sur Focillon ${ }^{24}$, j'ai évoqué longuement sa personnalité, son activité d'écrivain, même de porte-plume pour 
Gustave Geffroy, les mystères qui demeurent autour du tissu familial, etc., et guère son œuvre d'historien de l'art elle-même. Il faut aussi tâcher de projeter sur I'historien un éclairage qui ne soit pas nécessairement frontal.

Quant à l'enjeu politique de l'historiographie, il est évident que la psychologie et la psychanalyse, les aspects idéologiques, les outils conceptuels, l'écriture elle-même font nécessairement partie de toute recherche qui prétend étudier les discours sur l'art, autant que l'art lui-même. On ouvre alors la recherche sur des engagements personnels, que ce soit dans le cas de Louis Dimier ou de celui de Wilhelm Pinder !

L'histoire de l'art aujourd'hui, si on la considère d'un point de vue international, semble faire coexister des mondes qui, autrefois, seraient apparus comme incompatibles. D'un côté, les vieilles catégories subsistent - ainsi les quatre périodes académiques: antique, médiéval, moderne, contemporain - avec les vieilles oppositions - forme versus société ou contexte. D'un autre côté, s'est ouvert un vaste marché d'idées, de concepts, de méthodes, empruntant aux sciences humaines, à la French Theory, à l'anthropologie des mondes globalisés. Comment percevez-vous cette présentation du champ de l'histoire de l'art, à supposer que vous la trouviez pertinente?

Vous qui avez enseigné dans un lieu, le Collège de France, où l'innovation, la circulation des modèles d'une discipline à une autre, d'un pays à un autre, sont un principe constitutif, quelle leçon avez-vous tiré de votre expérience?

Roland Recht. Lorsque les anciennes catégories comme cette périodisation rigide subsistent, cela ne me gêne pas ; ce qui me gêne, c'est lorsqu'elles contribuent à régir un système de recrutement académique parfaitement obsolète. Dans ce cas, elles contrarient des efforts de recherches transversales, transhistoriques. Ce qui me paraît également très nuisible, $c^{\prime}$ est le clivage qui existe toujours entre l'université et les musées - il n'y a pas d'équivalent aux États-Unis ou en Allemagne. La circulation des conservateurs et des enseignants-chercheurs entre ces deux institutions, ou encore entre le CNRS et l'université, sont des nécessités absolues, pour le plus grand bénéfice de tous. Lorsque vous libérez ces passages, I'histoire de l'art s'aère également. Et le repli frileux de certains conservateurs sur leurs prés carrés en traitant les chercheurs comme autant d'intrus, ou le dédain que manifestent certains universitaires pour la pauvreté conceptuelle du travail des conservateurs, tout cela serait dépassé au profit d'une science appelée histoire de l'art. Elle serait alors bien plus solidement établie dans notre pays et ne considérerait pas comme une ingérence l'intérêt que manifeste un historien, un anthropologue ou un philosophe pour les images.

Mais j'aimerais souligner ceci : voir un tableau ou une sculpture sous le régime de l'image est une approche parfaitement légitime, mais tout à fait insuffisante pour I'historien de l'art. Il sait quel long chemin il convient encore de parcourir pour aborder ce tableau ou cette sculpture sous le régime que l'histoire de l'art instaure, par sa définition même : à savoir une connaissance des conditions matérielles et techniques dans lesquelles l'œuvre fut produite, une approche des forces psychiques qui sont à son origine, son inscription dans son historicité propre, qui n'est pas simplement le temps historique mais consiste aussi en une généalogie formelle. Et puis l'activité artistique suppose la vie sociale. L'histoire de l'art est donc aussi une science sociale, et les modalités qui régissent les relations entre l'artiste et la société ont un caractère spécifique. Voilà, me semble-t-il, ce qu'on pourrait attendre d'une histoire de l'art bien comprise.

Maintenant, pour en venir au Collège de France, il ne faut pas le considérer comme une abbaye de Thélème ! C'est l'endroit qui, dans le monde de la recherche, impose sans doute le plus de contraintes, d'obligations, tout simplement parce que 
le professeur est alors considéré, de l'intérieur comme de l'extérieur de l'institution, comme l' « ambassadeur » de sa discipline, ce qui est évidemment absurde. Ce sont les affinités personnelles entre collègues qui créent des circulations, pas l'institution. J'ai pu organiser un colloque avec le neurophysiologiste Alain Berthoz et deux autres avec le philosophe Jacques Bouveresse. Mais le fait que chaque professeur soit la plupart du temps le seul représentant de sa discipline au sein de la maison ne favorise pas forcément cette circulation. Et nous n'avons guère le temps d'aller dans les séminaires de nos collègues, ce qui constitue aussi une entrave.

Comment faites-vous cohabiter conscience historienne et conscience politique? En sous-texte, pouvez-vous rappeler la naissance de votre conscience politique, préciser la définition que vous appliqueriez à votre conscience historienne et donc revenir sur les négociations établies, le cas échéant, entre les deux?

Roland Recht. Pour les événements dont j'ai été le témoin direct ou indirect, je ne suis pas sûr de savoir bien faire la distinction entre ce qui a été pour moi un moment $d^{\prime}$ histoire ou le sentiment d'un éveil : né de parents très modestes dans une province de France occupée par l'Allemagne nazie, j'ai le souvenir d'attaques aériennes et d'abris, puis de la Libération. Souvenir aussi des guerres coloniales - I'Indochine et l'Algérie. Et puis I'année où je fus nommé assistant se termina par Mai 68, qui remettait en cause le magistère et avec lui le système sur lequel reposait la « fabrique des intellectuel $\mathrm{s}^{25}$ ». J'étais comme un apprenti nageur qui n'a plus pied. La conséquence a été une sorte d'ébranlement des certitudes, ce qui devait être le cas pour beaucoup d'entre nous : je vivais alors un dilemme entre un désir d'engagement moral et l'orientation esthétique que j'avais prise, ce que je considérais comme un dilemme entre engagement public et tour d'ivoire... J'ai songé un temps à abandonner l'histoire de l'art pour la sociologie ou l'ethnologie, mais pas pour I'histoire. Pourquoi ? Je pensais alors que l'histoire s'occupait du passé à partir de sources écrites ou orales qui n'étaient, après tout, que des sources secondaires, et que ce dont elles parlaient demeurerait à jamais insaisissable, et qu'au contraire, l'histoire de l'art s'empare de realia ; la sociologie ou l'ethnologie nous parlent d'hommes vivants et non de fantômes. Dans les représentations de la recherche que je me faisais, entrait la présence absolument nécessaire de realia. À l'opposé, I'histoire se tenait pour moi plus près de la « fiction », pour reprendre un mot de Michel de Certeau. C'était sans doute une vision bien naïve.

La distinction sur laquelle vous mettez l'accent me fait évidemment penser à Max Weber lorsqu'il définit le politique par rapport au savant : recherche scientifique et engagement politique ne sauraient être confondus. C'est une conception que l'on retrouve chez Marc Bloch et Lucien Febvre. L'historien doit non pas juger, mais comprendre les hommes du passé : la véritable recherche historique $\mathrm{n}^{\prime}$ instaure pas un tribunal afin de condamner telle ou telle figure d'il y a 50, 100 ou 300 ans. L'historien a pour objectif de chercher à comprendre, donc à expliquer. J'aime beaucoup cette distinction formulée par Marc Bloch et j'essaie de la faire mienne ${ }^{26}$. Et comprendre ne signifie pas porter des jugements de valeur, jugements que nous devons réserver à la chose publique. Or, la conscience politique peut altérer cet objectif, parce qu'elle oppose les affects au temps de la réflexion nécessaire au travail historien, elle a tendance à privilégier une analyse immédiate, en tous cas nécessairement courte, elle relève $d^{\prime}$ 'une heuristique de jugement. Mais ce sont deux consciences entre lesquelles il faudrait toujours chercher à ménager une certaine étanchéité.

La conscience historienne peut se manifester de plusieurs manières : par le souci d'une contextualisation qui permet de relativiser la position singulière de l'artiste 
4. Nam June Paik, Mac Ever's, 1989-1991, installation vidéo, achat, 1991, Strasbourg, musée d'Art moderne et contemporain. ou de l'œuvre ; par une grande prudence vis-à-vis de l'identification de modèles qui livreraient la clé de l'interprétation; par la remise en cause de notions usées à l'envi, comme celle $\mathrm{d}^{\prime}$ « influence »; par la prise en compte des décalages générationnels dans une perspective synchronique, etc. C'est aussi à une véritable conscience historienne que l'on doit la nécessité de partir du présent pour explorer le passé, d'où mon intérêt pour l'art contemporain ${ }^{27}$.

Et puis, je voudrais dire quelques mots sur la question de l'engagement. Pour I'historien (de l'art), il peut prendre plusieurs formes. L'historien peut jouer un rôle de conseiller, capable de conduire à la mise en œuvre des projets qu'il porte : l'exemple le plus illustre est sans doute celui d'André Chastel et de l'Inventaire des Monuments et richesses artistiques de la France, institutionnalisé par André Malraux. Cela suppose une relation de nature libérale entre le pouvoir et le " conseiller», et une telle relation n'est possible que dans une position quasi hégémonique des auteurs de ces projets $^{28}$. Cependant il existe d'autres formes d'engagement. L'historien (de l'art) peut, par exemple, ne pas se donner comme objectif I'accomplissement de ses idées et privilégier un regard libre, une expertise critique - c'est ce qui s'est passé lorsqu'en 1996, le président du Centre Pompidou, Jean-Jacques Aillagon, m'a confié une mission de réflexion sur l'avenir du Centre et du Musée national d'art moderne. Cette expertise peut aussi être appliquée à l'occasion d'articles de presse, ce que j'ai fait chaque mois pendant dix années lorsque je signais un éditorial dans le Journal des Arts (ou encore en publiant Penser le patrimoine, en 1999). Ma collaboration au journal Libération, à partir de 1993, elle, revêtait un aspect moins critique vis-à-vis des institutions car elle ne portait que sur des expositions et des livres ${ }^{29}$. Mais je pense que l'activité critique est quasi obligatoire pour quelqu'un qui est engagé dans la vie intellectuelle, il se doit d'être un observateur exerçant et faisant part d'une certaine vigilance.

Sans craindre d'arpenter l'immensité des sujets dits "grand angle", dans quelles mesures l'art et les cuvres sont-ils politiques? "Entre-t-on en art " comme on s'engage en politique? En quoi les œuvres d'art participent-elles du politique?

Pouvez-vous, sur ce point, préciser les formes d' "encartement " symbolique qui suscitent l'interrogation des intellectuels, et singulièrement des historiens de l'art?

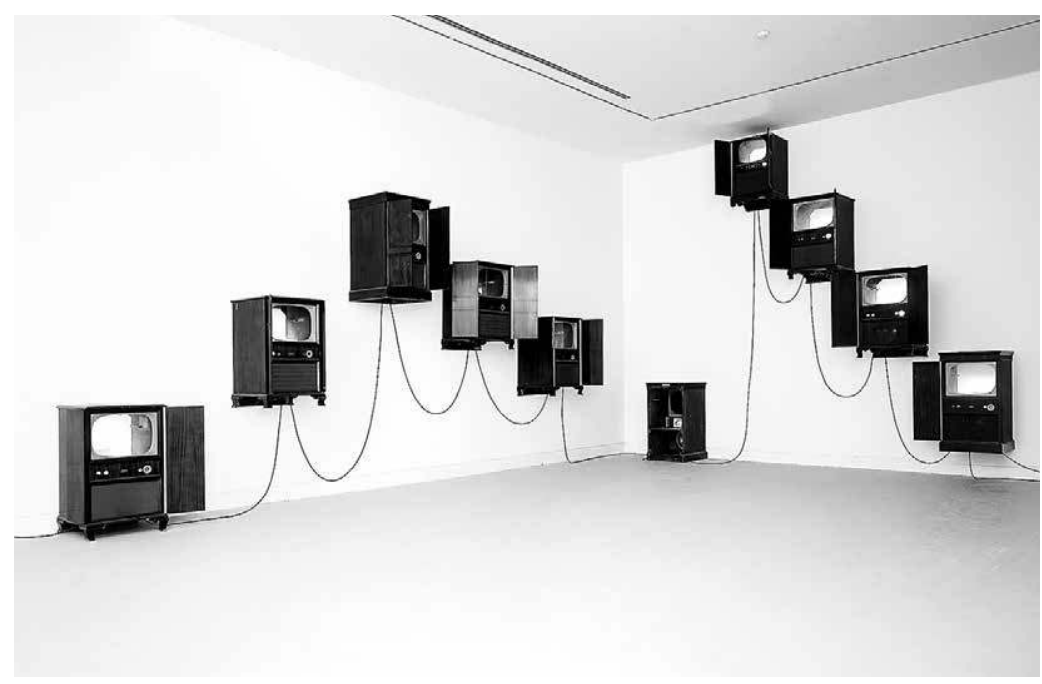

Roland Recht. C'est un truisme que de dire que toute œuvre d'art, quel que soit son auteur, est aussi un témoignage de son temps. Mais la création individuelle opère bien sûr un tri, aussi bien parmi ses possibilités expressives que parmi les préoccupations du moment, l'artiste cherchant plus ou moins obscurément à les mettre au jour. Tout à la fois consciente et inconsciente, la création artistique ne saurait se dérober systématiquement à tout ce qui relève de la vie sociale. Dans les jungles imaginées par le Douanier Rousseau, aussi inoffensives qu'un jardin d'acclimatation, surgissent des bêtes féroces qui faisaient peur au peintre lui-même. Qu'étaient 
censés exorciser ces félins ? Quel conflit intérieur se trouvait résolu (?) à chaque nouveau tableau ? Le petit employé de l'octroi devenu un peintre subitement adulé par les artistes les plus talentueux de son temps, voilà une belle intrigue. Car dans l'accomplissement d'une œuvre d'art se déploie toute la complexité d'une psyché dont les ressorts nous échapperont toujours. Nous ne travaillons que sur des bribes et nous ne formulons que des bribes d'interprétations.

L'hostilité que le public peut manifester à l'encontre de telle œuvre contemporaine est parfois le corrélat d'une position politique conservatrice ou même réactionnaire. Tout ce qui met en péril le ton dominant est compris comme une menace ou, au mieux, comme une facétie. Les avant-gardes du XXe siècle ont su jouer de ce rejet pour radicaliser encore davantage leurs programmes.

Mais il faut, je crois, opérer une distinction entre l'exposition temporaire qui peut plus facilement provoquer un scandale (comme celle de Harald Szeemann à Berne en 1969, "Quand les attitudes deviennent formes ${ }^{30} »$ ), et la collection d'un musée qui, même si elle contient des œuvres radicales, tend à unifier, à l'intérieur d'une historicité commune, des œuvres qui relèvent de syntaxes bien différentes. Le travail unificateur du musée et la légitimité qu'il confère aux œuvres exposées « dépolitisent » en quelque sorte leurs portées respectives. Alors, montrer dans une exposition temporaire des œuvres d'art moderne est en soi un acte politique, du moins dans la mesure où le concept qu'elles illustrent ou sous-tendent a un contenu qui ne se soumet pas au sens commun. Une même œuvre peut choquer dans tel contexte et se trouver banalisée dans tel autre. Je n'ai jamais cherché à choquer, mais à trouver le ton juste pour souligner la pertinence du propos : de toute façon, une fois le choix $d^{\prime}$ un artiste prononcé, il travaille librement.

Comment un intellectuel engagé construit-il une politique culturelle à l'échelle locale? Comment un savant, entré dans l'administration, devient-il un "décideur politique"? Quels modes opératoires sont les siens?

Roland Recht. Pour comprendre la position qui était la mienne en tant que directeur des Musées de Strasbourg, il faut signaler que j'ai été conjointement nommé par la Direction des musées de France et le maire de Strasbourg. Du $1^{\text {er }}$ janvier 1986 au 30 septembre 1993, date de ma démission de ce poste, j'ai pu construire une politique culturelle, fort de cette double tutelle. Connu comme spécialiste de l'architecture médiévale, j'avais cependant la charge des huit musées, chacun disposant d'un conservateur, couvrant toutes les périodes et toutes les formes d'art : un pied dans le patrimoine historique, l'autre dans la modernité puisqu'on $\mathrm{m}^{\prime}$ avait immédiatement chargé du projet d'un nouveau musée, consacré à l'art moderne et contemporain. Si dans les années 1960 c'était dans la construction de maisons de la Culture que les villes faisaient un investissement symbolique, vingt années plus tard, c'était dans celle de musées d'art moderne.

En faisant acquérir dans les années 1985-1990 une installation de Nam June Paik (fig. 4), des peintures de Schönebeck et de Baselitz, ou encore une œuvre de
5. Giovanni Battista Tiepolo, Zéphir et Flore, vers 1751-1753, Strasbourg, musée des BeauxArts, donation O. Kaufmann et F. Schlageter en 1987.

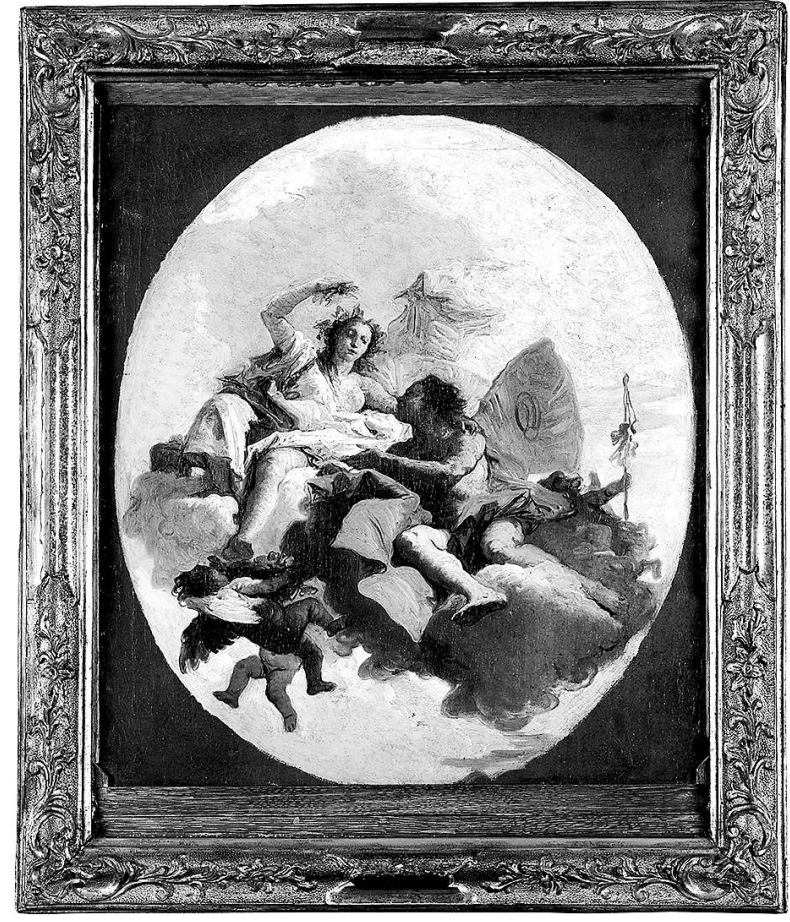



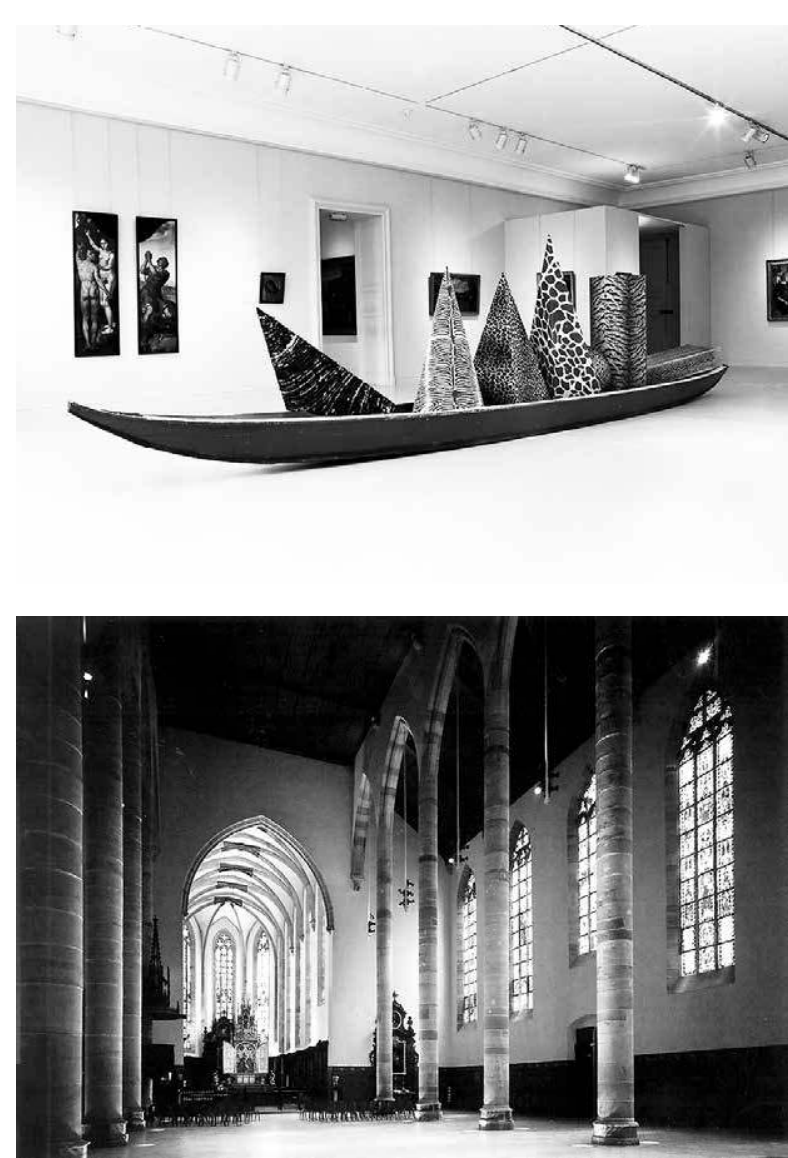

6. Claudio Parmiggiani, Zoo Geometrico, 1969 (Amsterdam, Stedelijk Museum), présenté dans l'exposition "Saturne en Europe », à Strasbourg (ici installé au musée des Beaux-Arts) en 1988.

7. Vue de la nef vers le chœur, église des Dominicains de Colmar.
Broodthears, je ne choisissais pas la facilité dans une ville où le patrimoine ancien tenait une place primordiale. Certes, susciter une importante donation de peintures baroques $^{31}$ (fig. 5), tout comme acquérir des œuvres de Arp et de Schwitters, dans les mêmes années, compensait en quelque sorte ces audaces aux yeux du public et des édiles. Mais afin de montrer la portée des œuvres contemporaines, j'organisais en 1988 une exposition intitulée "Saturne en Europe. Regards sur la modernités2 ${ }^{2}$. Elle thématisait la mélancolie saturnienne à partir d'œuvres mettant en scène une écriture allégorique faite de traces, de fragments, de ruines, du déploiement au sol, etc ${ }^{33}$ (fig. 6). C'était aussi, choisi délibérément, un propos mettant en perspective la notion d'installation, un dispositif en partie aléatoire articulé entre l'architecture du lieu et les objets.

Or, dans "Saturne en Europe », plusieurs œuvres $d$ 'artistes contemporains étaient montrées non pas dans une salle d'exposition, mais au musée des Beaux-arts, au musée des Arts décoratifs, au musée de l'œuvre NotreDame, donc au cœur des collections anciennes. Dans la mesure où il n'est pas excessif de parler d'un geste " politique », il était là. En 1988, une telle confrontation était exceptionnelle, dans le public elle choquait les tenants de I'orthodoxie d'un goût normatif : lorsqu'ils entrent dans un musée, ils savent ce qu'ils vont y trouver, une illustration, une confirmation de leur goût. Toute intrusion d'un corps étranger et anachronique est jugée comme une atteinte inacceptable à l'ordre esthétique qu'ils prônent, c'est-à-dire à l'ordre tout court. Or, tout au contraire, de telles confrontations proclament une certaine continuité de l'histoire, mais selon des registres grammaticaux différents. La présence d'un anachronisme renforce l'impact visuel de l'ancien comme du nouveau, et incite à une plus grande acuité. C'est une formidable contribution à la formation du regard, mais elle dépend et du choix que l'on a fait de l'artiste et surtout du sens de l'espace que possède l'artiste lui-même.

Vos premiers travaux concernent l'histoire de l'architecture religieuse gothique ${ }^{34}$. Qu'est-ce qui vous a conduit à étendre vos intérêts à d'autres périodes? Comment vos approches ont-elles évolué?

Roland Recht. Mon intérêt pour l'architecture a été d'abord celui que l'historien porte aux monuments du Moyen Âge : la majorité d'entre eux nécessite une critique d'authenticité supposant un examen approfondi et l'établissement d'une chronologie absolue ou du moins relative. Celle-ci est tributaire d'une contextualisation ainsi que d'une étude comparative. Voilà, en raccourci, le chemin que parcourt I'historien de l'architecture médiévale. Mais son travail devient encore plus intéressant lorsqu'il détecte les modèles et leur circulation : il est alors obligé d'aborder des problèmes de géographie, d'histoire des institutions religieuses et politiques, de circulation, d'économie du travail, etc. Mes premiers travaux, concentrés autour du Rhin supérieur entre le XIII et le XVe siècle, insistent sur l'opposition entre l'architecture des collégiales ou des églises paroissiales tributaires du modèle de la cathédrale de Strasbourg, l'un des tout premiers chantiers 
de l'Empire, et les églises qu'érigent les ordres mendiants - dominicain (fig. 7) et franciscain. Cette opposition se révèle parfois insuffisante lorsque l'on constate que des formes particulièrement raffinées, contraires à l'esprit de simplicité prôné initialement par ces ordres, sont empruntées par eux au modèle cathédral. Pourtant les deux typologies conservent des caractères suffisamment stables pour qu'elles puissent, jusqu'à un certain point, servir de modèle conceptuel pour I'historien.

Si on étudie l'architecture d'une période donnée, on ne peut pas rester indifférent à celle d'autres périodes. Cependant, à partir de la Renaissance, l'existence des traités et l'abondance des sources qui nous livrent le nom des architectes modifient radicalement notre perception des monuments par rapport à ceux du Moyen Âge. Mais ce qui m’a surtout intéressé, c'est le rapport que l'architecture moderne, même contemporaine, entretient avec celle du Moyen Âge parce qu'on observe alors comment certains architectes cherchent à s'émanciper de la tradition vitruvienne, classique donc, en se tournant vers des modèles gothiques. Au XXe siècle encore, nous voyons des architectes comme Mies van der Rohe ou Le Corbusier porter un jugement sur la valeur, positive ou négative, que détient à leurs yeux I'architecture gothique. Mon intérêt pour ces questions recoupe celui que je manifeste pour l'historiographie puisque nous nous situons là sur le vaste terrain de ce que la théorie littéraire appelle la « réception ».

Lorsque I'on étudie l'architecture du Moyen Âge, on se retrouve fatalement un jour au XIX ${ }^{\mathrm{e}}$ siècle qui non seulement a "inventé " le Moyen Âge, mais qui est aujourd'hui un objet historiographique tout à fait majeur. À la suite de la Révolution française, une histoire allant de la préhistoire jusqu'aux temps modernes en incluant le Moyen Âge s'est peu à peu construite, avec ses témoins matériels, artistiques et architecturaux. Le corpus patrimonial du Moyen Âge - celui de la littérature et de I'art - va consolider avec force le récit national. Ce qui m'intéresse, ce n'est pas le néo-gothique, la paraphrase du gothique, qui est en soi un champ d'étude tout à fait légitime, mais plutôt comment le gothique a stimulé la pensée architecturale des temps modernes : ce passage-là nous autorise à considérer différemment l'architecture gothique elle-même ${ }^{35}$ (fig. 8).

Quel est votre regard sur l'histoire de l'architecture en France et à l'étranger aujourd'hui ? Connait-elle une crise au sein des universités françaises? Quels sont ses rapports avec l'histoire de l'art?

Roland Recht. En France, à la différence de l'Italie, nous n'avons jamais eu beaucoup de spécialistes de l'architecture. Les premières chaires d'histoire de l'art créées à la Sorbonne sont destinées à un généraliste (Henry Lemonnier) ou à un iconographe (Émile Mâle), au Collège de France à un spécialiste de la peinture (Charles Blanc). Toujours au XIXe siècle, à l'École du Louvre, les enseignements portent sur les collections du musée et ne traitent qu'accessoirement d'architecture. Arcisse de Caumont s'appuie sur la tradition des antiquaires anglais tandis qu'en Allemagne, Franz Kugler est le premier historien à envisager une histoire systématique de l'architecture universelle au milieu du XIXe siècle, déjà. II faut aussi rappeler qu'en France, l'importance accordée à l'étude des monuments anciens d'Italie dans l'enseignement de l'École des beauxarts a privilégié l'Antiquité, et on connaît la difficulté avec laquelle y fut introduite assez tard l'étude des monuments médiévaux. C'est la création de la Commission des monuments historiques, sous la monarchie de Juillet, qui a définitivement consacré 
un champ d'étude (et d'enseignement) qui s'étend sur toutes les périodes de l'histoire de l'architecture en France. Se dessinent ainsi différentes inflexions qui marqueront d'une façon durable les générations suivantes.

Je fais ce rapide rappel historique pour faire mieux comprendre la situation actuelle que je considère comme irréversible. Comme je l'ai dit, l'éducation à l'architecture et à la ville est une des grandes oubliées de l'enseignement public ${ }^{36}$. Aucun ministère de l'Enseignement supérieur et de la Recherche scientifique n'a compris l'importance de cette éducation, ni le rôle qu'elle pourrait jouer dans la formation de l'esprit et de la sensibilité individuelle. Quant au ministère de la Culture, il préfère mettre l'accent sur des « coups » médiatiques comme les Journées du Patrimoine, dont les statistiques de fréquentation n'ont évidemment aucune valeur : on mobilise les publics durant un weekend alors que les musées et les monuments les moins emblématiques restent vides la plupart du temps. Je n'ai pas besoin de souligner qu'une éducation de ce type aurait des conséquences politiques non négligeables. Éveiller chez le jeune élève une attention à son environnement architectural et urbain constituerait une tâche sans doute difficile pour les maîtres, même formés, mais pourrait faire progressivement des usagers de la ville et de l'architecture de véritables acteurs de leur environnement quotidien, lequel ne serait alors plus livré aux seuls architectes et aux édiles.

Je pense que la crise que traverse l'enseignement de l'histoire de l'architecture aujourd'hui pourrait recevoir un début de solution si l'enseignement primaire et secondaire faisait une place à l'environnement architectural et urbain. J'ajouterai que l'évolution plutôt favorable à l'histoire de l'architecture que connaissent les écoles d'architecture aujourd'hui, où le niveau de l'enseignement théorique et historique s'est nettement amélioré, est un facteur positif.

D'une façon générale, l'importance démesurée que prend aujourd'hui l'étude de l'image au sein même de l'histoire de l'art ne fait que creuser un fossé entre elle et l'histoire de l'architecture qui est pourtant sa sœur jumelle. II est d'ailleurs tout à fait éloquent de constater que les philosophes, les historiens ou les anthropologues, lorsqu'ils font un pas de côté, le font toujours du côté des images, jamais du côté de I'architecture. Les images se montrent sans doute plus dociles...

Vous vous êtes particulièrement intéressé au dessin d'architecture et, d'une façon plus générale, à la représentation comme à l'expérience de l'espace. Pouvez-vous en expliquer l'importance?

Roland Recht. L'architecture a toujours été pour moi un objet étonnant, et par là-même passionnant, pour deux raisons majeures qui enjambent l'une et l'autre les délimitations historiques. Elle est d'abord, et je dirai par définition, indescriptible. Je me considère comme un nominaliste, c'est-à-dire que, pour moi, une œuvre d'art a besoin du langage pour exister. C'est grâce à lui qu'elle peut faire l'objet d'un récit - I' « histoire » de l'art - et ce qu'elle éveille en nous d'émotions ou de jugements esthétiques ne peut être communiqué que par le langage. Simultanément, nous devons faire l'aveu d'une impuissance à décrire l'œuvre d'art. Des philosophes comme Max Dessoir ou des historiens comme Erwin Panofsky ont bien pris en compte cette aporie en pointant le fait que toute description est un regard biaisé, qu'elle implique une orientation de l'esprit, au fond qu'elle est déjà « interprétation ». On peut aussi substituer à une description une œuvre poétique : c'est ce que prônait le philosophe Karl-Philip Moritz dans sa critique de Winckelmann, en disant que seul un équivalent poétique était en mesure de rendre compte de la beauté d'une œuvre plastique. On pourrait aussi citer l'ekphrasis exemplaire de Diderot. Mais qui a su parler aussi remarquablement 
de l'architecture ? Tant qu'il s'agit de mettre des mots sur les objets architecturaux, tout va bien. C'est la tension entre ces objets qui est intéressante, ce sont leurs interrelations qui façonnent un espace.

L'architecture ne s'avère pas seulement indescriptible, elle se dérobe à toute construction narrative, sauf sous la forme d'équivalents. À mes yeux, il n'y a pas d'approche plus suggestive de l'espace que des récits littéraires qui mettent en œuvre une logique combinatoire, comme ceux $d^{\prime}$ 'Italo Calvino - par exemple ses Villes invisibles de 1972 - ou encore La Bibliothèque de Babel, où Jorge Luis Borges imagine un dispositif qui repose sur la combinatoire et l'ars memoriae des anciens. Pour moi, tout espace dans l'architecture, mais aussi dans la nature, paysagère ou non, est, grâce aux objets architecturaux (ou naturels) qui le scandent, une sorte de théâtre de mémoire ${ }^{37}$. La mémoire repose sur un déploiement spatial.

Je disais que je voyais deux raisons à mon attirance pour l'architecture. La seconde est que l'architecture est le contenant de tous les contenants. Le livre, la sculpture, la peinture, les arts décoratifs, la musique trouvent leurs lieux d'élection dans I'architecture où elles déploient leurs pouvoirs imaginants. Temple ou cathédrale, villa ou palais, elle est par excellence le lieu où l'homme va à la rencontre des images, et de l'art. Tout aboutit à l'architecture. Et nous sommes tellement inclus nous-mêmes en elle, que nous ne lui accordons que peu d'importance comme nous attachons peu $d^{\prime}$ importance à nos vêtements, une fois choisis.

Et c c'est là que je voudrais consacrer quelque attention à $\mathrm{I}^{\prime}$ « espace ${ }^{38} »$. C'est un mot-valise tout comme le mot « image ». Nous l'employons en lui attribuant les significations les plus diverses. Je me limiterai, en tant qu'historien, à une définition élémentaire : I'ensemble des intervalles créés par les objets architecturaux. Et par objets architecturaux, j'entends bien sûr les supports, les couvertures, les murs, les ouvertures. En parlant ainsi, je ne rends pas compte de toutes les qualités sensibles qui s'attachent à I'espace. Il peut donner un sentiment d'oppression ou au contraire de grande amplitude, etc. En histoire de l'art, il importe de ne pas adopter une définition "substantialiste » de l'espace, comme celle de Focillon - " l'espace de l'art est matière plastique et changeante » écrit-il dans Vie des Formes -, ni formaliste, comme celle de Wölfflin - chaque «style » historique modèlerait son propre espace. Le livre de Bruno Zevi, qui cherche à définir d'une façon très nuancée ce qu'est l'espace architectural, a joué un rôle décisif dans l'intérêt pour l'architecture chez les gens de ma génération : Zevi ${ }^{39}$ était une figure d'intellectuel italien tout à fait typique, à la fois architecte, historien et critique, au ton incisif, militant, dirais-je. Et puis j'ai beaucoup appris auprès du grand médiéviste qui a été mon professeur, Louis Grodecki, qui possédait un sens de l'espace tout à fait exceptionnel. Je dirai enfin que c'est mon intérêt pour l'espace architectural qui a aussi porté mon attention vers les installations des artistes des années 1960-1970.

Quand on s'intéresse à l'espace architectural, le musée devient un objet passionnant. Sa configuration détermine des lieux où les objets témoins du temps historique, tout comme dans l'analyse combinatoire des mathématiciens, ne peuvent être soumis qu'à un nombre limité de combinaisons spatiales.

À la différence des objets architecturaux, l'espace ne peut pas être représenté par le dessin. L'expérience que nous en avons est fondée sur la déambulation, les mouvements du corps, les changements de points de vue, mais aussi la lumière, l'odeur, les sons qui s'y propagent. II doit y avoir nécessairement entre la pensée, d'une part, et ce qui va devenir le dessin des objets architecturaux, d'autre part, une interpolation qui suppose une conscience de l'espace reposant sur nos expériences antérieures. La phénoménologie de Maurice Merleau-Ponty inclut ces deux questions - celle de l'interaction entre la conscience et le corps, et celle de la projection de formes hors du $\operatorname{corps}^{40}$. 


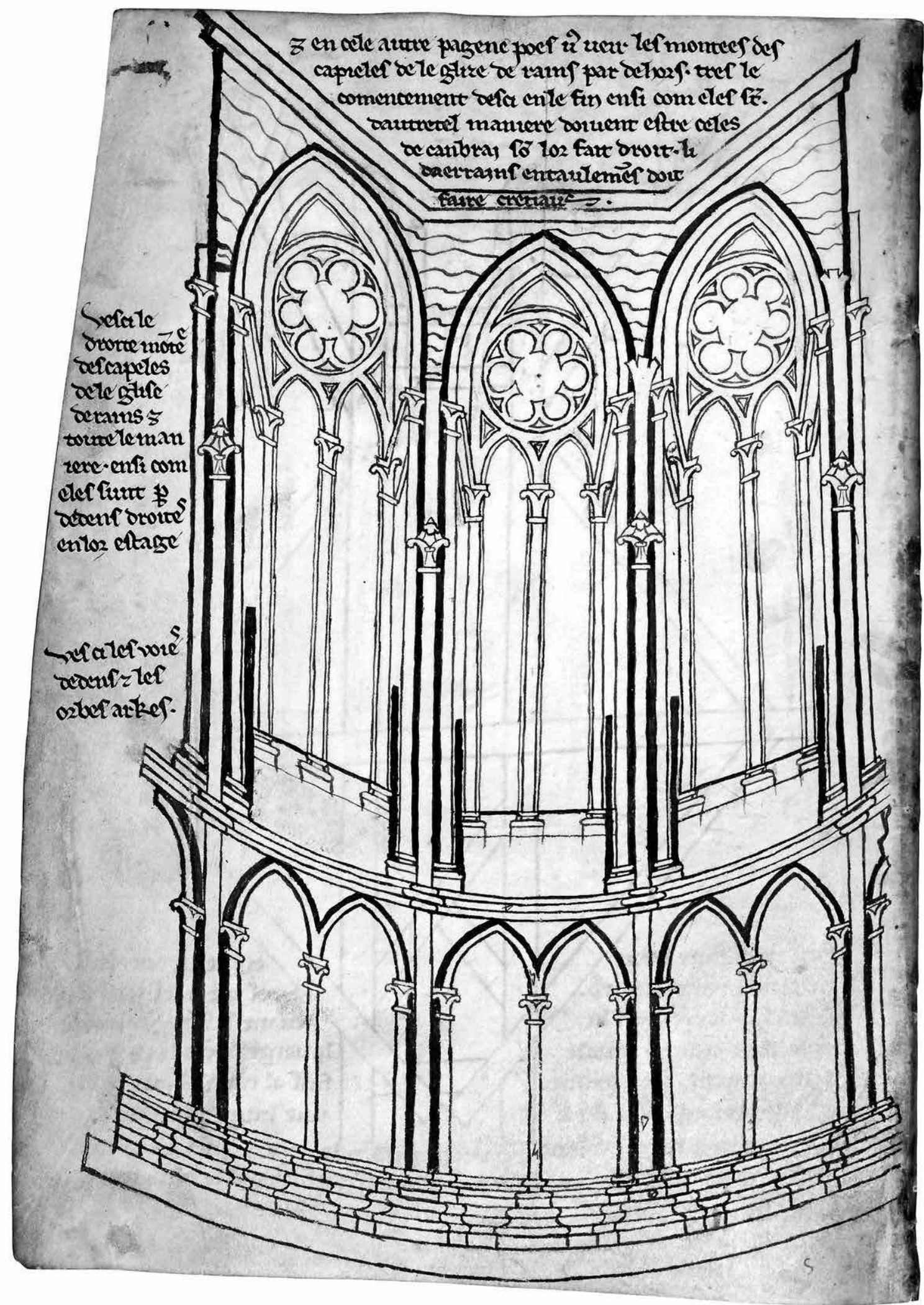


D'où mon intérêt pour le dessin d'architecture (fig. 9). Dans l'absolu, une architecture élémentaire, même quelque peu élaborée, ne nécessite pas qu'on en dessine le plan et l'élévation. Dans l'architecture civile rurale ou urbaine d'autrefois, il faut imaginer des modes opératoires relativement simples qui permettent de passer d'un plan tracé au sol à l'élévation, sans outils conceptuels très pointus. Il en était sans doute de même pour l'architecture romane. Mais à un moment donné, l'établissement de plans, d'élévations, de sections s'est avéré nécessaire afin de rendre possible l'intelligibilité et la réalisation de structures de plus en plus élaborées : c'était le cas de I'architecture gothique. À partir de ces dessins remontant, pour les plus anciens conservés, au milieu du XIII siècle, on peut entrer dans une opération mentale très complexe.

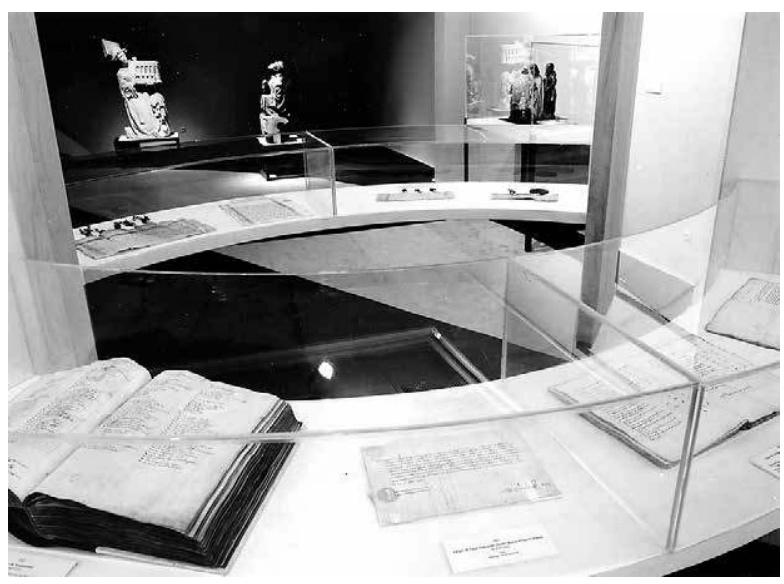
Ce qui m'intéressait alors, c'était d'assister à la genèse de tout un processus de codification à l'aide duquel un architecte dessine simplement en un trait des formes en deux dimensions afin de suggérer des formes en trois dimensions. L'invention de ces dessins n'a pas seulement une signification épistémologique, elle marque aussi une mutation essentielle dans la sociogenèse du chantier : I'activité de I'architecte change, et son statut social en même temps. On assiste alors à la naissance de la figure moderne de l'architecte. Dorénavant, il consacrera plus de temps à ce travail qu'à la surveillance du chantier, qu'il va déléguer. Ces dessins vont avoir une double fonction : technique, je l'ai dit, et symbolique. Car il s'agit de faire aussi d'un dessin de façade, par sa dimension et par le soin mis à son tracé, une œuvre d'art qui devra séduire le commanditaire : la puissance épiscopale en représentation. Le dessin est également le véhicule des inventions formelles qui vont être transmises par le chantier à d'autres formes d'art: c'est cette fonction que j'ai voulu, entre autres, illustrer dans mon exposition sur "Les bâtisseurs de cathédrales », en $1989^{41}$ (fig. 10). Enfin, dans les années 1960, mon intérêt pour le dessin d'architecture a été en quelque sorte stimulé par l'importance que lui accorde un architecte comme Aldo Rossi en tant qu'outil théorique et critique, mais aussi historique puisqu'il travaille sur la ville en tant que superposition de strates.

Le rapport entre conception et perception des formes et des volumes a-t-il été transformé par les technologies informatiques? L'usage de la "réalité augmentée " au sein des monuments vous semble-t-elle utile (dans le contexte de l'évolution des politiques et des idées patrimoniales)?

Roland Recht. Il est évident que les restitutions en trois dimensions des monuments anciens mettent à la disposition des chercheurs, des étudiants, des outils qui enrichissent le processus cognitif et qui modifient notre recours à l'imagination. C'est notre perception de la représentation tridimensionnelle qui change lorsque nous voyons un monument de l'intérieur et que nous pouvons le faire tourner sur un axe virtuel de 90 ou de $360^{\circ}$. Reconstituer un monument en ruine devient ainsi une opération relativement aisée et d'un intérêt archéologique indéniable.

La réalité augmentée est une autre chose. Certaines de ses applications, dans le domaine de la chirurgie par exemple, vont permettre des avancées considérables. Dans le domaine patrimonial, nous assistons au début d'une redéfinition du « culte moderne des monuments ». Par exemple, les travaux du centre de recherche Inria de Bordeaux développent le programme Manao selon le principe dit de « la lampe torche magique »

9. Villard de Honnecourt, vue intérieure d'une chapelle de la cathédrale de Reims, vers 1220-1230, encre sur parchemin, Paris, BnF, département des Manuscrits, Fr. 19093, fol. $30 v^{\circ}$.

10. Vue de l'exposition "Les Bâtisseurs de cathédrales ", à Strasbourg, Ancienne Douane, en 1989. 
qui permettra de voir un objet placé dans une vitrine de musée sans le toucher, mais avec plus de détails - avec sa polychromie primitive - que ne peut en voir le visiteur aujourd'hui. Le Allard Pierson Museum, aux Pays-Bas, a utilisé ce procédé. C'est un exemple parmi d'autres augmentant fondamentalement l'expérience cognitive, et qui donne une idée de ce que pourra devenir l'exploitation du patrimoine monumental dans I'avenir. Mais pour en revenir à Merleau-Ponty et à sa Phénoménologie de la perception, comment le corps et notre cerveau réagissent-ils à ces machines qui annulent la distance ? Comment le corps immobile vit-il ses déplacements et mouvements virtuels ? Le mathématicien Henri Poincaré a, dès le début du siècle dernier, insisté sur l'importance du mouvement dans la perception de l'espace : I'homme astreint à l'immobilité ne connaîtrait tout simplement pas l'espace. Plus d'un siècle plus tard, avec les importants progrès accomplis, grâce aux neurosciences, dans la connaissance du cerveau et des réseaux cérébraux qui rendent compte de cette perception, que pouvons-nous dire sur la différence entre la perception, par un même sujet, d'un espace réel et celle $d^{\prime}$ un espace " réellement augmenté » ? Je ne saurai bien sûr répondre à cette question. Mais je reprendrai volontiers la phrase de Georges Pérec que j'avais citée dans mon introduction au symposium organisé avec Alain Berthoz au Collège de France : «Vivre, c'est passer d'un espace à un autre, en essayant le plus possible de ne pas se cogner ${ }^{42}$. » Si cela ne vous semble pas trop trivial, ce sera aussi ma conclusion. 
1. Roland Recht, À quoi sert l'histoire de l'art? Entretien avec Claire Barbillon, Paris, 2006.

2. Laurent Baridon, L'imaginaire scientifique de Viollet-le-Duc, Paris, 1996.

3. Roland Recht, Philippe Sénéchal, Claire Barbillon, François-René Martin (dir.), Histoire

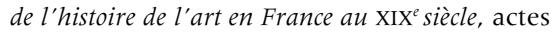
de colloque (Paris, Institut national d'histoire de l'art/Collège de France, 2004), Paris, 2008.

4. Agnès Callu (dir.), Autopsie du musée : études de cas, 1880-2010, Roland Recht (préface), Paris, 2016.

5. Agnès Callu, Roland Recht, L'historien de l'art : conversations dans l'atelier, Strasbourg [à paraître en 2018].

6. Voir, en particulier, Roland Recht, Daniel Russo, "Interview avec Roland Recht", dans Bulletin du centre d'études médiévales d'Auxerre | BUCEMA, $\mathrm{n}^{\circ} 18,2,2014$, mis en ligne le 28 décembre 2014 : http://cem.revues.org/13556; DOI : 10.4000/ cem.13556 (consulté le 14 février 2017).

7. Par exemple Martin Warnke, Wilhelm Schlink, Enrico Castelnuovo, Maurizio Ghelardi...

8. Roland Recht, Nicolas de Leyde et la sculpture à Strasbourg (1460-1525), Strasbourg, 1987 ; Idem, "Nicolas de Leyde et son temps ", dans Nicolas de Leyde, sculpteur $d u X V^{e}$ siècle. Un regard moderne, Cécile Dupeux, Roland Recht, Stefan Roller (dir.), cat. exp. (Strasbourg, musée de l'œuvre Notre-Dame, 2012), Strasbourg, 2012, p. 19-25. Idem, "La rhétorique formelle de Claus Sluter, sculpteur du duc de Bourgogne ", dans Martin Büchsel, Peter Schmidt (dir.), Das Porträt vor der Erfindung des Porträts, Mayence, 2003, p. 205-217, Idem, « “...Le redéploiement douloureux de l'être sur lui-même." Le combat de Rodin ", dans L'invention de l'auvre. Rodin et les Ambassadeurs, Dominique Viéville (dir.), cat. exp. (Paris, musée Rodin, 2011), Arles/ Paris, 2011, p. 45-61.

9. Voir tout récemment l'essai accompagnant l'édition de l'Atlas Mnémosyne, d'Aby Warburg, Paris, 2012.

10. Roland Recht, Le dessin d'architecture : origine et fonctions, Paris, 1995.

11. À Dijon, en 1983-1984.

12. Roland Recht, La Lettre de Humboldt : du jardin paysager au daguerréotype (1989), Paris, 2006.

13. Idem, Le croire et le voir : l'art des cathédrales, XII-XVesiècle, Paris, 1999.

14. Idem, Point de fuite. Les images des images des images. Essais critiques sur l'art actuel, 1987-2007, Paris, 2009.

15. À mon grand regret, l'historiographie de la cathédrale de Strasbourg entre 1770 (Goethe !) et nos jours, enjeu idéologique considérable, n'a jamais été entreprise. Elle tourne parfois (encore dans les années 1970 chez un savant allemand, Reinhard Liess, dans nombre de ses publications relatives aux dessins d'architecture pour la cathédrale de Strasbourg) autour de la figure singulière (et mythique) de Erwin von Steinbach, considéré comme l'auteur de sa prodigieuse façade, avec des accents nationalistes qu'on croyait définitivement révolus. Dans les mêmes années, une monographie de ce monument due à un savant suisse contient maints passages caractérisés par la déconsidération des travaux allemands les plus récents.

16. Carl Justi, Winckelmann: sein Leben, seine Werke und seine Zeitgenossen, Leipzig, 18661872, 3 vol. ; Wilhelm Waetzoldt, Deutsche Kunsthistoriker von Sandrart bis Justi, Leipzig, 1921-1924, 2 vol.

17. En particulier le livre de Hans Tietze, Methode der Kunstgeschichte, Leipzig, 1913.

18. Notamment Aloïs Riegl (Sandro Scarrocchia, Oltre la storia dell'arte: Alois Riegl, vita e opere di un protagonista della cultura viennese, Milan, 2006), Georg Dehio (Peter Betthausen, Georg Dehio. Ein deutscher Kunsthistoriker, Berlin, 2004), Wilhelm Pinder (Marlite Halbertsma, Wilhelm Pinder en de Duitse kunstgeschiedenis, Groningen, 1985), Erwin Panofsky (Silvia Ferretti, Il demone della memoria. Simbolo e tempo storico in Warburg, Cassirer, Panofsky, Gênes, 1984), Wilhelm Vöge, Adolph Goldschmidt (Kathryn Brush, The Shaping of Art History. Wilhelm Vöge, Adolph Goldschmidt and the Study of Medieval Art, Cambridge, 1996), Hugo von Tschudi (Barbara Paul, Hugo von Tschudi und die moderne französische Kunst im Deutschen Kaiserreich, Mayence, 1993), etc. Les références aux travaux sur Jacob Burckhardt et Aby Warburg sont trop nombreuses pour être citées ici.

19. Aux éditions l'écarquillé, Paris. Sont parus jusqu'ici : Miroir de faille. À Rome avec Giordano Bruno et Edouard Manet, 1928-1929, Maurizio Ghelardi (éd.), Paris, 2011 ; L'Atlas Mnémosyne. Avec un essai de Roland Recht, Paris, 2012 ; Fragments sur l'expression, Susanne Müller (éd.), Paris, 2015.

20. Sur ce plan ils rejoignaient les positions de Charles-Victor Langlois et Charles Seignobos dans l'Introduction aux études historiques (1898), Paris, 1992, qui n'accordaient qu'une place mineure à l'histoire de l'art, leur intérêt pour les témoins matériels du passé se concentrant sur l'archéologie.

21. On sent bien dans le compte rendu que Febvre fait du livre de son collègue strasbourgeois Pierre Francastel, L'humanisme roman. Critiques des théories sur l'art du XI 'eiècle en France, Rodez, 1942, qu'il ne voit l'intérêt de l'histoire 
de l'art qu'en tant que sœur cadette de l'histoire, une Cendrillon assise en bout de table, pour reprendre l'une de ses métaphores.

22. Certes, il ne faut pas négliger le travail accompli à partir de 1900 par Henri Berr avec la Revue de synthèse historique : par exemple, le tome 28, de 1914, principalement consacré à l'histoire de l'art avec, entre autres, des contributions de Hans Tietze et August Schmarsow.

23. La brusque éclosion d'ouvrages généraux sur l'histoire de l'art dans les années 1990 semble s'être réduite à une compétition entre éditeurs : aucun d'entre eux n'a cru nécessaire d'enrichir ces manuels d'introductions méthodologiques ou historiographiques (voir mon éditorial : "Manuels et histoires générales de l'art ", dans Revue de l'art, n 124, 1999 , p. 5-11).

24. Roland Recht, "Henri Focillon, Rome et Piranèse. Portrait du poète sous le signe de Saturne ", dans La Vie des formes : Henri Focillon et les arts, Christian Briend, Alice Thomine (dir.), cat. exp. (Lyon, musée des Beaux-Arts, 2004), Gand, 2004, p. 55-67.

25. Louis Bodin, Les intellectuels existent-ils?, Paris, 1997, p. 51 ; voir aussi Agnès Callu (dir.), Le Mai 68 des historiens : entre identités narratives et histoire orale, actes de colloque (Paris, Collège de France, 2008), Jacques Revel (préface), Daniel Roche (postface), Villeneuve d'Ascq, 2010.

26. Dans le chap. IV de l'Apologie pour l'Histoire ou Métier d'historien, Paris, 1949.

27. J'ai réuni un certain nombre d'essais dans Recht, 2009, cité n. 14.

28. Cette hégémonie a pris fin après 1968, lorsque le projet d'un enseignement de l'histoire de l'art porté par les historiens de l'art Jacques Thuillier et André Chastel a capoté, en faveur d'un enseignement des arts plastiques défendu par l'esthéticien Bernard Teyssèdre.

29. J'ai remis ma démission au Journal des Arts, auquel j'avais collaboré sur l'invitation de Philippe Régnier, tout comme j'ai cessé ma collaboration à Libération où m'avaient convié Hervé Gauville et Antoine de Gaudemar, chaque fois lorsque mon entière liberté d'expression s'est trouvée remise en cause par leurs successeurs.

30. When Attitudes Become Form: Works, Concepts, Processes, Situations, Information. Live in your Head, Harald Szeemann (dir.), cat. exp (Kunsthalle Bern, 1969), Berne, 1969.

31. Il s'agit de la donation Othon Kaufmann et François Schlageter faite au musée des Beaux-Arts de Strasbourg en 1987 et les années suivantes.
32. Saturne en Europe, Roland Recht, Françoise Ducros (dir.), cat. exp. (Strasbourg, Ancienne Douane/musée des Beaux-Arts/musée des Arts décoratifs, 1988), Strasbourg, 1988.

33. Les artistes étaient Joseph Beuys, Christian Boltanski, Marcel Broodthears, Gérard CollinThiébaut, Ian Hamilton Finlay, Thomas Huber, Gerhard Merz, Iannis Kounellis, Anselm Kiefer, Claudio Parmiggiani, Giuseppe Penone, Sarkis.

34. L'Alsace gothique 1300-1360. Études d'architecture religieuse, Colmar, 1974.

35. Plusieurs essais anciens sur ce thème sont à présent regroupés dans Revoir le Moyen Âge. La pensée gothique et son héritage, Paris, 2016.

36. Recht, 2006, cité n. 1.

37. Voir surtout Frances A. Yates, L'art de la mémoire, Paris, 1975 [éd. orig. The Art of Memory, Chicago, 1966] et un ouvrage antérieur de Paolo Rossi, Clavis Universalis. Arts de la mémoire, logique combinatoire et langue universelle de Lulle à Leibniz, Grenoble, 1993 [éd. orig. Clavis universalis. Arte della memoria e logica combinatoria da Lullo a Leibniz, Bologne, 1960], injustement négligé.

38. Voir Alain Berthoz, Roland Recht (dir.), Les espaces de l'homme, actes de colloque (Paris, $3^{\text {e }}$ Symposium annuel du Collège de France, 2003), Paris, 2005.

39. Bruno Zevi, Apprendre à voir l'architecture, Paris, 1959 [éd. orig. : Saper vedere l'architettura, Turin, 1948] : c'est un ouvrage que je n'ai cessé de recommander à mes étudiants, en raison de son ton très stimulant.

40. Maurice Merleau-Ponty, Phénoménologie de la perception, Paris, 1945.

41. À l'occasion de cette exposition est paru un catalogue : Les bâtisseurs des cathédrales gothiques, Roland Recht (dir.), cat. exp. (Strasbourg, Ancienne Douane, 1989), Strasbourg, 1989.

42. Berthoz, Recht, 2005, cités n. 38 